\title{
Primary Osteosarcoma of the Maxillary Sinus: An Exceptional Presentation
}

\section{Imane Mbarki*, Samia Hajar Touimi, Sanae Elmajjaoui, Tayeb Kebdani, Hanane Elkacemi, Noureddine Benjaafar}

Department of Radiation Oncology, National Institute of Oncology, Mohammed V University, Rabat, Morocco

Correspondence to: Imane Mbarki, Department of Radiation Oncology, National Institute of Oncology, Mohammed V University, Rabat, Morocco. Received date: May 21, 2021; Accepted date: June 2, 2021; Published date: June 8, 2021

Citation: Mbarki I, Touimi SH, Elmajjaoui S, et al. (2021) Primary Osteosarcoma of the Maxillary Sinus: An Exceptional Presentation. J Med Res Surg 2(3): pp. 1-3. doi: $10.52916 /$ jmrs214047

Copyright: @2021 Mbarki I, et al. This is an open-access article distributed under the terms of the Creative Commons Attribution License, which permits unrestricted use, distribution and reproduction in any medium, provided the original author and source are credited.

\section{ABSTRACT}

Osteosarcoma is a common primary malignant tumor of long bones in which mesenchymal cells produce osteoid, affecting mainly young adults. Head and neck localization is rare, moreover at the level of the maxillary sinus. The certainty diagnosis is based on the clinical CT and histopathological findings. This histological type is associated with a low risk of metastasis. Early diagnosis and wide surgical resection of the tumor are the most important determinant factors of prognosis. In this article, we report a case where radiotherapy was used.

\section{Keywords:}

Osteosarcoma, Maxillary Sinus, Surgery, Radiotherapy, Chemotherapy

\section{Abbreviations:}

CT: Computed Tomography; Gy: Gray; MRI: Magnetic Resonance Imaging; VMAT: Volumetric Modulated Arc Therapy; OS: Overall Survival; DFS: Disease-Free Survival

\section{Introduction}

Osteosarcoma is rare cancer. It is the second most common primary malignant tumors of bone, after multiple myeloma, accounting for $<0.2 \%$ of all cancers. It is the most common malignant bone tumor in children and adolescent [1]. Typical sites of osteosarcoma are long bones. Primary maxillary sinus involvement is very rare. Characterized by a bimodal distribution, Osteosarcoma of head and neck is seen in the second and third decade. Maxillary sinus osteosarcoma tends to be painful, symptoms depending on the importance of tumor size and extension to proximal organs. CT is an essential tool in the diagnosis, it highlights the aggressive radiological characteristics of the lesion: erosion of the cortical bone, soft tissue involvement, periosteal reaction. Treatment includes complete resection when surgically possible, with associated radiotherapy or chemotherapy. Exclusive radiotherapy is reserved for locally advanced forms surgically recused.

\section{Case Report}

A 37-years-old female patient was referred to the Department of Radiation Oncology at the National Institute of Oncology by an otolaryngologist due to right jugal swelling progressively increasing in size. It is was a young patient with no contributory past medical history, complaining of a palpable mass in the right maxillary region for four months. Clinical examination finds facial asymmetry, with swelling of the right maxillary region associated with ipsilateral exophthalmia. The cutaneous plan was respected. No lymphadenopathy was found in the cervical lymph node levels. The ophthalmologic examination found no alterations of ocular motility or reflexes. Visual acuity was $8 / 10$ in the right eye and 10/10 in the left eye.

A facial massif CT with contraste injection (Figure 1) showed invasive tissue filling of the right maxillary sinus causing osteolysis of the maxillary and malar bones with exophthalmia.

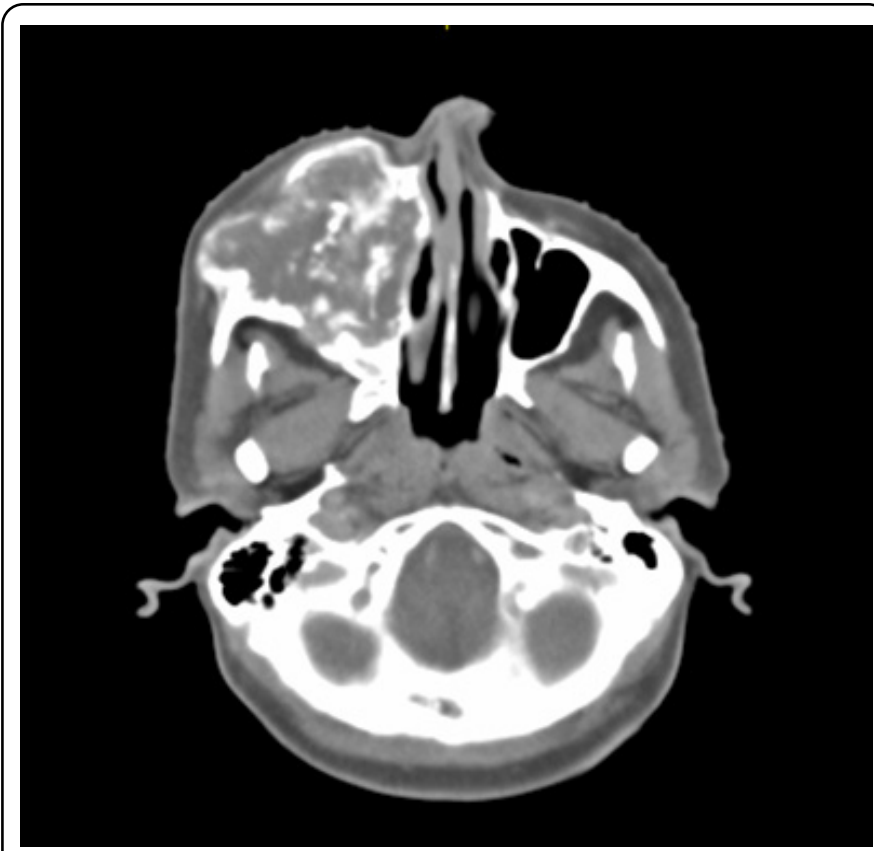

Figure 1: CT showing the tumor process leading to lysis of the right maxillary sinus.

A MRI (Figure 2) was performed revealed a lytic tissue tumor process centred on the right maxillary sinus, with irregular contours, heterogeneously enhanced after injection of gadolinium, measuring $66 \times 54 \times 59 \mathrm{~mm}$. This process bombs in the ipsilateral nasal fossa; it invades the right infra-temporal fossa, the soft cheekbones and the jawbone, which is lysed. It comes into contact with the orbital floor. No intra-orbital or endocranial extension. Absence of cervical lymphadenopathy. The thoracic-abdominopelvic CT does not detect secondary lesions at distance. A biopsy was performed of the lesion. The microscopic study and immune-histochemical analysis lead to the diagnosis of high-grade osteosarcoma. After a multidisciplinary board, the decision taken consisted of neoadjuvant chemotherapy to decrease the size of the tumor, facilitate surgical resection and increase the chances 


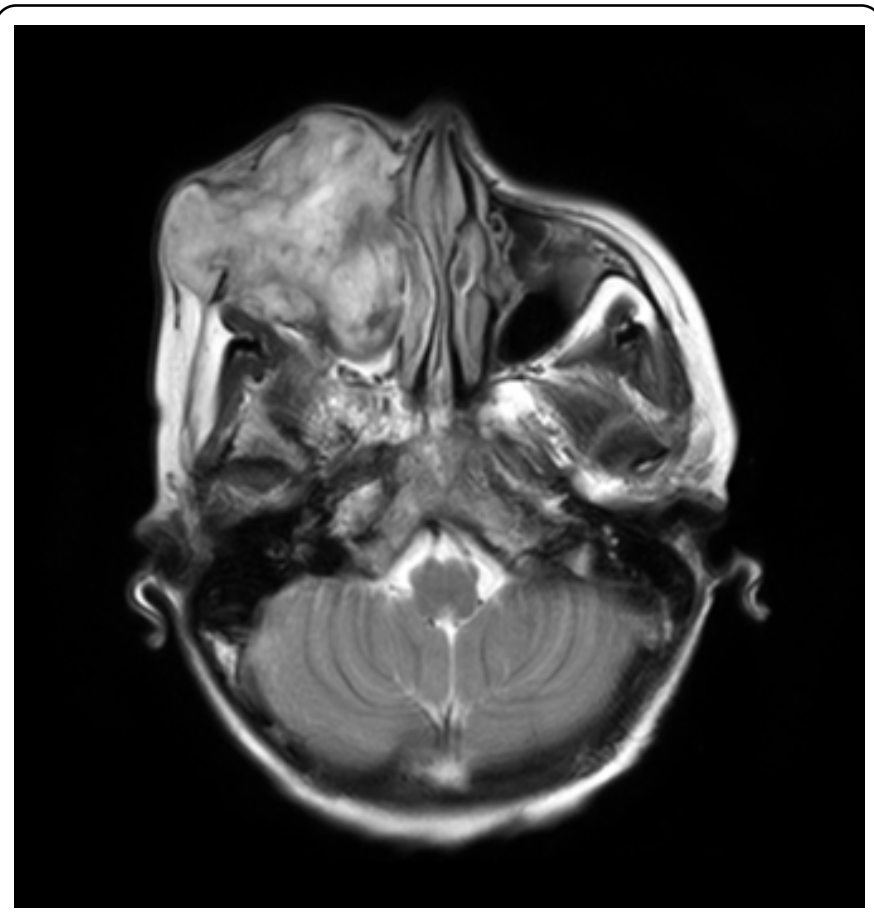

Figure 2: MRI showing a filling of the right maxillary sinus by an aggressive tumor process.

of obtaining negative surgical limits. The patient received 4 cycles of chemotherapy (adriblastine, cisplatin and ifosfamide). A good clinical response has been observed after cycles of chemotherapy. However, the Patient was surgically recused. Given the aggressive nature of this histologic type, the decision of radiotherapy was laid. The patient received external beam radiation therapy. The patient received conformational external radiotherapy, a dose of $70 \mathrm{~Gy}$ in VMAT technique in $2 \mathrm{~Gy} /$ fraction, 5 sessions per week.

\section{Discussion}

Osteosarcoma of head and neck is a rare entity of all osteosarcomas, the incidence reporting in studies is between $0.5-8 \%[2,3]$. It has a bimodal age distribution, with a peak incidence in the second and third decades of life and another smaller peak in adults older than 65 years of age. An association with other osseous abnormalities should be searched when the diagnosis of osteosarcoma is made earlier than the second decade or after the cessation of skeletal growth. Indeed, osteosarcoma can arise in the context of a genetic predisposition or underlying abnormalities such as Paget disease, bone infarcts, osteomyelitis [4] or fibrous dysplasia. Later in life, it can present as secondary tumors after radiation therapy [5]. There is a slight male predilection, with a ratio of 1.2:1 [6].

Osteosarcoma occurs most frequently in the lower long bones. The craniofacial region is not a common site for the development of this histological type. Osteosarcomas of nasosinus cavities are very rare account for $0.5-1 \%$ of all tumors occurring in nasosinus cavities [7]. There is a high rate of local recurrence and a reduced risk of distant metastasis for sarcomas of head and neck compared to the osteosarcoma of the long bones, which makes the importance of early diagnosis. The most common symptoms of osteosarcoma of nasosinus cavities are mass or swelling, pain, and nasal obstruction. Other less common complaints include epistaxis or eye displacement. Radiographic and histologic analysis are mandatory for the definitive diagnostic and therapeutic decision. The three main radiological signs are bone formation, bone destruction and periosteal reaction. The most common radiographic feature is a mixed appearance with irregular margins. Perforation of the cortical plates and invasion to the surrounding soft tissues are common [8].

The maxillary sinus is an air-filled cavity, and therefore even a small lesion can be easily detected by radiological tools, which highlight the importance of radiology in the early diagnosis of these lesions before the invasion of nearby structures. Radiologic features of maxillary sinus lesions help to determine their origin and behavior to get close to a more definitive diagnosis. In our case, the right maxillary sinus cavity was invaded and filled with the lesion. Biopsy of the tumor should be performed to confirm the diagnosis and differentiate from other bone lesions. Microscopically, the essential criterion of osteosarcoma is the direct production of osteoids or immature bone by tumoral cells. Three more common histologic variants including osteoblastic, fibroblastic and chondroblastic, which considered the subtype with higher prevalence in the head and neck region and is associated with a better prognosis [9].

The main prognostic factors of osteosarcoma of nasosinus cavities are tumor size, histological grade, and resectability at the time of diagnosis [10]. Early diagnosis and negative surgical margins are important to obtain local control and improve the prognoses of patients with head and neck osteosarcoma [11]. As it is the case for other skeletal cancer, surgery is the standard treatment also in head and neck region [12]. The surgical technique consists of aggressive resection and advanced reconstruction. The single most important factor for definite an optimal carcinological resection is clear margins. Nevertheless, this goal is difficult to achieve because of the complexity and anatomic limitation of the maxillofacial region [13]. Local recurrences and intracranial invasion have long been reported as the major causes of treatment failure due to incomplete neoplasm resection [14].

A multidisciplinary strategy by adding chemotherapy and/or radiotherapy to surgery has demonstrated improved survival in locoregionally advanced head and neck cancer. Chemotherapy reduces tumor's size, ameliorate surgical resection, improve local control and reduce distance metastases. The interest of using adjuvant radiotherapy is to decrease the risk of locoregional relapses. A systemic review on 201 patients from 20 uncontrolled series had evaluated the role of chemotherapy [15]. 60 patients received chemotherapy (neo-adjuvant regimen for 18 patients, and Adjuvant regimen for 42 patients), surgery performed in 180 patients. The 5-year OS and ProgressionFree Survival (PFS) in this group of patients undergoing multimodal therapy were 80 and $75 \%$, respectively. Therefore, chemotherapy significantly improved survival when combined with radical surgery.

Recently, chemotherapy directed by targeted nanoparticulate drug delivery system represents a promising approach for osteosarcoma treatment, using RGD peptide-installed doxorubicin-loaded biodegradable polymeric micelle, or by 2-Methoxyestradio, it's a physiological metabolite of 17 
$\beta$-estradiol, cause cell cycle arrest and apoptosis in osteosarcoma cells $[16,17]$.

The role of radiotherapy has been demonstrated in a study conducted by Guadagnolo et al [18], who evaluated the role of radiotherapy in 119 patients affected by osteosarcoma of craniofacial region. 92 patients underwent surgery alone, and 27 cases, surgery was followed by radiotherapy. Based on the analysis of resection margin, OS (80 vs. $31 \%$ ) and DFS (80 vs. $35 \%$ ) were superior in patients with positive or uncertain margins treated by surgery and radiotherapy compared to patients treated by surgery alone. This high-risk group is inclined to get the best results, while no advantage is expected for patients with negative margins.

For patients who are not candidates for surgery because of choice or associated comorbidities, radiotherapy is an alternative for local control. A prescription dose of $60 \mathrm{~Gy}$ in 2-Gy fractions is typically used for microscopically involved margins, whereas $66 \mathrm{~Gy}$ is used for macroscopic residual disease and 70 Gy is used for inoperable tumors. The radiation technique used, either three-dimensional conformal or intensity-modulated radiation therapy should be tailored to the individual patient. Dose to uninvolved organs should be minimized to prevent late organ dysfunction, as should the integral dose to minimize the risk of secondary malignancy. Patients with poor performance status or seriously ill should be offered optimal supportive care in order to control symptoms and preserve the quality of life.

\section{Conclusion}

The rarely of this antomo-clinical entity and de complexity of the maxillofacial region makes osteosarcoma of nasosinus cavity challenging to manage. A collaboration between the anatomopathologist, the otolaryngologist the oncologist and the radiotherapist is essential for better management of patients

Acknowledgment: We thank the Radiotherapy department and our radiotherapist's colleagues at the National Institute of Oncology of Rabat who provided care and support for the patient.

Disclosures: The authors report no conflict of interest concerning the case in this paper.

Consent: Written informed consent was obtained from our patient for publication of this case report and accompanying images.

\section{References}

1. Stiller CA, Bielack SS, Jundt G, et al. (2006) Bone tumours in European children and adolescents, 1978-1997. Report from the Automated Childhood Cancer Information System project. Eur J Cancer 42(13): pp. 2124.

2. Bozdogan Arpaci R, Yuyucu Karabulut Y, Kara T, et al. (2015) Osteosarcoma of the Sinonasal Cavity in a Child. Oral Surg Oral Medi Oral Pathol Oral Radiol 119(3): pp. e127.
3. Gadwal SR, Gannon FH, Fanburg-Smith JC, et al. (2001) Primary osteosarcoma of the head and neck in pediatric patients: a clinicopathologic study of 22 cases with a review of the literature. Cancer 91(3): 598- 605.

4. Kirby EJ Zhou HH, Louis Morales L Jr (2011) Primary osteosarcoma of the skull. J Craniofac Surg 22(6): 2399-2405.

5. August M, Magennis P, Dewitt D (1997) Osteogenic sarcoma of the jaws: factors influencing prognosis. Int J Oral Maxillofac Surg 26(3): pp. 198-204.

6. Gurney JG, Swensen AR, Bulterys M (2011) SEER pediatric monograph. http://seer.cancer.gov/publications/childhood/ bone.pdf.

7. Wenger DE, Sundaram M, Unni KK, et al. (2002) Microscopic correlation of radiographically disparate appearing well differentiated osteosarcoma. Skeletal Radiol 31(8): pp. 488-492.

8. Doval DC, Kumar RV, Kannan V, et al. (1997) Osteosarcoma of the jaw bones. Br J Oral Maxillofac Surg 35:357- 62 .

9. Junior AT, de Abreu Alves F, Pinto CA, et al. (2003) Clinicopathological and immunohistochemical analysis of twenty five head and neck osteosarcomas. Oral Oncol 39(5):52130.

10. Gadwal SR, Gannon FH, Fanburg-Smith JC, et al. (2001) Primary osteosarcoma of the head and neck in pediatric patients: a clinicopathologic study of 22 cases with a review of the literature. Cancer 91(3): pp. 598-605.

11. Gorsky M, Epstein JB (2000) Craniofacial osseous and chondromatous sarcomas in British Columbia: A review of 34 cases. Oral Oncol 36(1): pp. 27-31.

12. BA Guadagnolo, GK Zagars, AK Raymond, et al. (2009) Osteosarcoma of the jaw/craniofacial region: outcomes after multimodality treatmen. Cancer 115(14): pp. 3262-3270.

13. Jasnau S, Meyer U, Potratz J, et al. (2008) Craniofacial osteosarcoma experience of the cooperative German-AustrianSwiss osteosarcoma study group. Oral Oncol 44(3): pp. 286-294.

14. Geopfert H, Raymond AK, Spires JR (1990) Osteosarcoma of the head and neck. Cancer Bull 42(3): pp. 347-354.

15. Smeele LE, Kostense PJ, van der Waal I, et al. (1997) Effect of chemotherapy on survival of craniofacial osteosarcoma: a systematic review of 201 patients. J Clin Oncol 15(1): pp. 363367.

16. Fang Z, Sun Y, Xiao H, et al. (2017) Targeted osteosarcoma chemotherapy using RGD peptide-installed doxorubicin-loaded biodegradable polymeric micelle. Biomed Pharmacother 85(1): pp. 160-168.

17. Gorska M, Kuban-Jankowska A, Zmijewski $M$, et al. (2015) 2-methoxyestradiol (DNA strand breaks induced by nuclear hijacking of neuronal NOS as an anti-cancer effect of 2-methoxyestradiol. Oncotarget 6(2): 15449-63.

18. Guadagnolo BA, Ashleigh Guadagnolo B, Zagars GK, et al. (2009) Osteosarcoma of the jaw/craniofacial region. Cancer 115(14): pp. 3262-3270. 